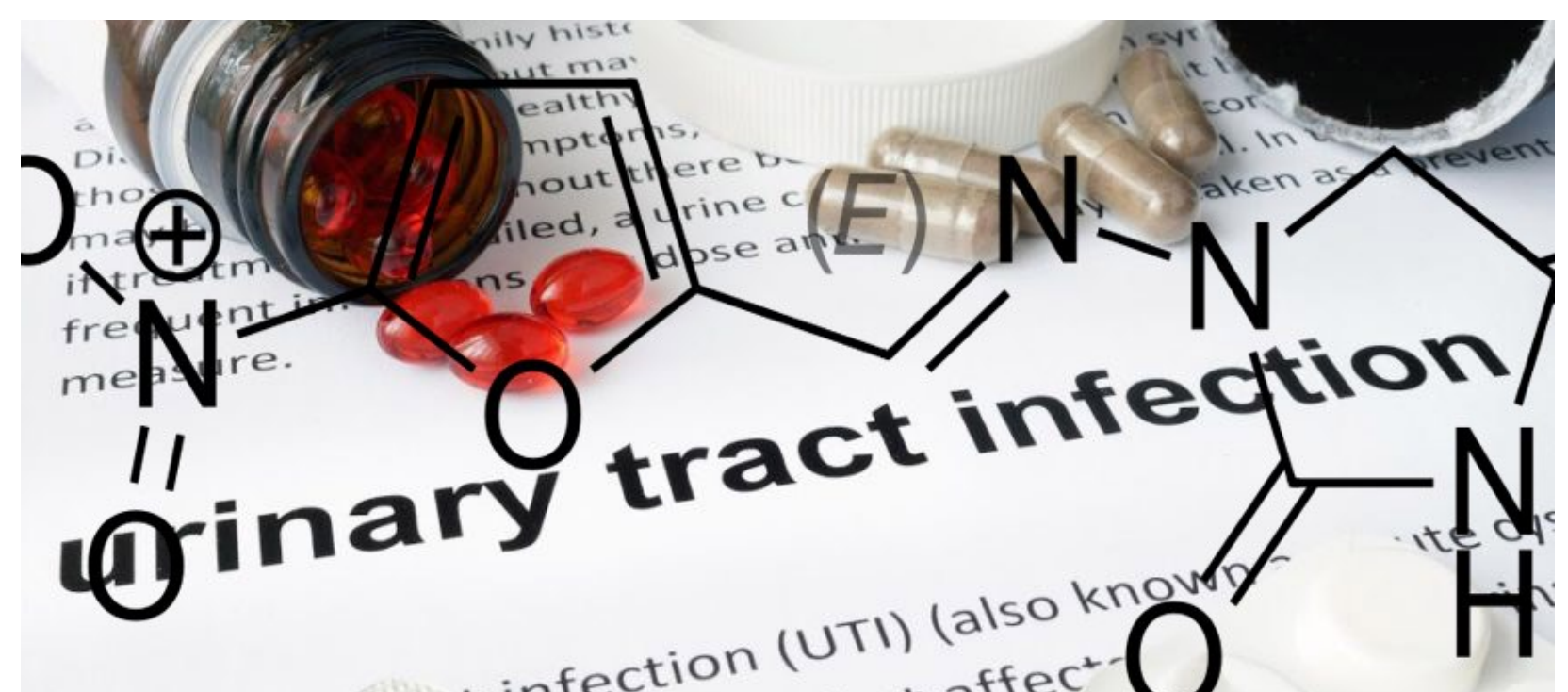

A urinary

infecti

- infection (UTI) (also kng

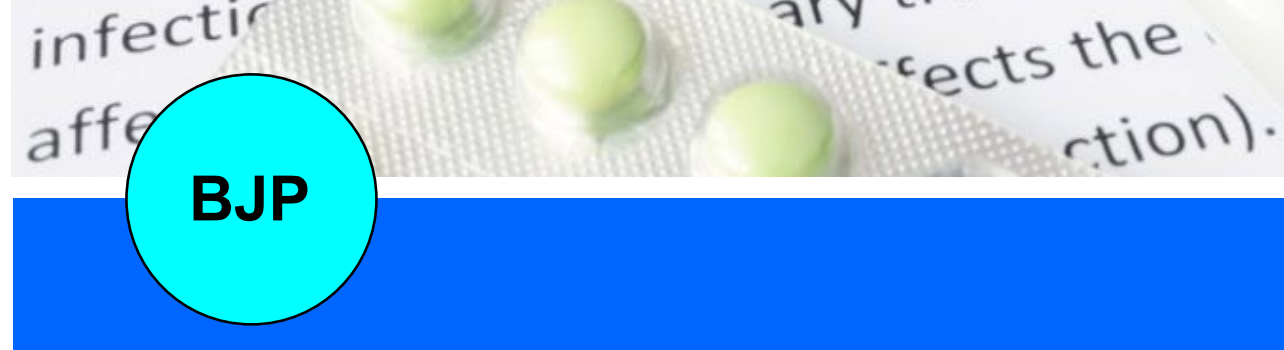

Bangladesh Journal of Pharmacology

Research Article

Reintroduction of nitrofurantoin in the treatment of uncomplicated urinary tract infection 


\title{
Reintroduction of nitrofurantoin in the treatment of uncomplicated urinary tract infection
}

\author{
Sabiha Mahboob and Md. Sayedur Rahman \\ Department of Pharmacology, Faculty of Basic Science and Paraclinical Science, Bangabandhu Sheikh Mujib \\ Medical University, Shahbag, Dhaka, Bangladesh.
}

\begin{tabular}{|c|c|}
\hline \multicolumn{2}{|l|}{ Article Info } \\
\hline Received: & 18 June 2021 \\
\hline Accepted: & 2 July 2021 \\
\hline Available Online: & 3 July 2021 \\
\hline \multicolumn{2}{|c|}{ DOI: 10.3329/bjp.v16i3.54481 } \\
\hline \multicolumn{2}{|c|}{$\begin{array}{l}\text { Cite this article: } \\
\text { Shabnam S, Rahman MS. Reintroduc- } \\
\text { tion of nitrofurantoin in the treatment } \\
\text { of uncomplicated urinary tract infec- } \\
\text { tion. Bangladesh J Pharmacol. 2021; } \\
\text { 16: 87-90. }\end{array}$} \\
\hline
\end{tabular}

\begin{abstract}
The present study was designed to reintroduce an older antimicrobial nitrofurantoin to treat uncomplicated urinary tract infection and to explore the relationship between the reintroduction and corresponding shift in sensitivity pattern of the microbes. The microbial sensitivity against ciprofloxacin before intervention was $46.6 \%$, which significantly increased after intervention $(51.8 \%)$. Treatment with nitrofurantoin resulted in a total clinical cure rate of $80.6 \%$, of which rate of clinical cure achieved in expected time was $66.1 \%$ and delayed was $14.5 \%$. The rate of total clinical cure with nitrofurantoin corresponded closely with the proportion of urinary isolates sensitive to nitrofurantoin. i.e., $86.3 \%$. This preliminary study revealed that revival might be an effective strategy to increase the sensitivity and to delay the emergence of resistance.
\end{abstract}

\section{Introduction}

Emergence of resistant bacterial infection has become a startling issue and is one of the greatest threats for human health (WHO, 2014). It is estimated that antimicrobial resistance could cause 10 million deaths per year by 2050 (Kraker et a;., 2016). There is a direct relationship between antimicrobial consumption and development of resistance (Ventola 2015), which was explained by the selection pressure (Laxminarayan et al., 2013). In spite of prediction, the resistance among microbes emerged within a decade of introduction of penicillin (Farrell, 2009). After penicillin, discovery of number of novel antimicrobials allowed us to combat resistance (Aminov, 2010). However, the promotional activities of the pharmaceutical industries contributed adversely to increased use of newer medicines, particularly antimicrobials (Das and Rahman, 2010; Johora and Rahman, 2018). At some point of time, innovation of novel antimicrobials appeared inadequate to contain the alarming spread of antimicrobial resistance (Laxminarayan et al., 2013). Multidisciplinary approach had become the mainstay for containment of antimicrobial resistance, recycling of older antimicrobials is one of the approaches (Cassir et al., 2014).

Decreasing consumption of antimicrobial is followed by increase in the susceptibility (Austin et al., 2010). A steady decrease in consumption of antimicrobial results in change in the sensitivity pattern (Gottesman et al. 2009). Modifying the prescription is one of the approaches attempted for reducing antimicrobial consumption and thereby resistance (Ventola et al., 2015). However, the knowledge and perception about antimicrobial among Bangladeshi future physicians appeared as a major barrier to rational prescribing of antimicrobial (Ferdoush et al., 2016). Though bringing change in the prescription of the physicians is very challenging, there are few established evidence-based methods to modify the antimicrobial prescribing, which in turn reduces resistance (CDC, 2017). Formulation of antimicrobial guideline based on laboratory evidence at 
institutional level was found to be effective to certain extent (Sultana and Rahman, 2017). Using older antibiotics rationally could contribute positively to preserve the sensitivity of the newer one's for a longer period (Cassir et al., 2014). The idea of prescribing nitrofurantoin in patients of uncomplicated urinary tract infection was reintroduced by implementing academic detailing to the prescribers and the effect of this reintroduction on the sensitivity pattern of microbes against ciprofloxacin was observed.

\section{Materials and Methods}

The present observational analytical study was conducted in Combined Military Hospital, Dhaka, Bangladesh. Culture-sensitivity data was collected from Armed Forces Institute of Pathology, Dhaka. The study was conducted from May 2018 to January 2019, though the initial work started since September 2017. Military Hospital was chosen purposively to conduct the study in closed community settings in order to minimize the influence of other factors on sensitivity of microbes. Combined Military Hospital, Dhaka was selected as this is the largest military hospital of the country with excellent microbiology laboratory facility.

\section{Academic detailing}

Sensitivity data of the urinary culture (extending from January to May 2018) was collected from Armed Forces Institute of Pathology, Dhaka and was compiled to demonstrate the sensitivity pattern. An academic detailing package including printed materials and predesigned verbal content was designed (Islam et al., 2007; Das and Rahman, 2010) illustrating the local sensitivity trend and the international guideline of uncomplicated urinary tract infection. The educational intervention in the form of academic detailing was implemented in June 2018. The researcher volunteered

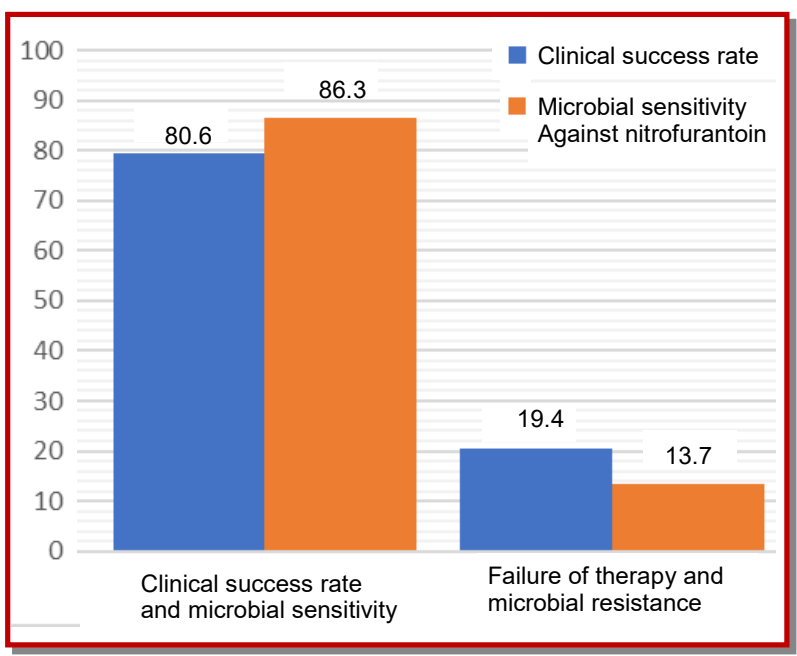

Figure 1: Clinical care rate and microbial sensitivity against nitrofurantoin ( $\mathrm{Y}$ axis is expressed as percentage) to provide any information requested by the prescribers.

\section{Enrolment of subject}

Immediately after diagnosing any patient suffering from uncomplicated urinary tract infection, the prescribers contacted the researcher. The researcher talked to the patient and if the patient fulfils the study criteria, they were explained and approached to participate in the research and if agreed, an informed written consent was obtained. Telephone numbers were obtained from enrolled patients to communicate them in order to evaluate the clinical outcome. Prescriptions were documented by capturing picture. If clinical improvement did not occur within 72 hours, the prescribers switched to their preferred alternative. Prescription data were collected from July 2018 to November 2018.

\section{Analysing sensitivity}

Sensitivity data after the educational intervention were collected from Armed Forces Institute of Pathology, Dhaka and were analysed and compiled in Microsoft excel worksheet.

\section{Analysing clinical cure}

After completion of treatment, all patients were contacted over telephone and the clinical cure and failure of therapy was evaluated using telephonic conversation compilation Form. These data were recorded, stored and finally analysed.

\section{Results}

Microbial sensitivity against ciprofloxacin and nitrofurantoin before and after the intervention was calculated. Total 2,256 and 2,153 urinary samples were analysed respectively before and after the intervention for sensitivity against ciprofloxacin. Among which $46.6 \%$ $(1,051$ out of 2,256$)$ were sensitive to ciprofloxacin before the intervention, which was increased to $51.8 \%$ $(1,115$ out of 2,153$)$ after the intervention. In case of nitrofurantoin, total 2,183 and 2,075 urinary samples were analysed respectively before and after the intervention. Out of which, $84.0 \%(1,834$ out of 2,183$)$ were sensitive to nitrofurantoin before intervention, which was increased to $86.3 \%(1,791$ out of 2,075$)$ after the intervention.

The sensitivity to ciprofloxacin after intervention [51.79 $(1,115 / 2,153)]$ increased significantly $(p<0.001)$ than before $[46.58(1,051 / 2,256)]$. The sensitivity to nitrofurantoin after intervention $86.3(1,791 / 2,075)]$ increased significantly than before $[84.0(1,834 / 2,183)]$.

\section{Clinical cure rate and sensitivity of urinary microbial isolates to nitrofurantoin}

Total 62 patients were enrolled as study subjects. Among them, clinical cure rate was assessed through 
information obtained through telephonic conversation. Total clinical cure rate was $80.6 \%$ (50 out of 62) and failure of therapy was $19.4 \%$ (12 out of 62 ). Among the patients get cured, clinical cure at expected time was $66.1 \%$ (41 out of 62 ) and remaining, i.e., $14.5 \%$ (9 out of 62) had delayed clinical cure.

Figure 1 represents the clinical cure rate and sensitivity of urinary microbial isolates to nitrofurantoin after the intervention. Here, the clinical cure rate was similar to that of the sensitivity of urinary microbial isolates to nitrofurantoin and therapeutic failure was similar to that of microbial resistance.

\section{Discussion}

Antimicrobial resistance is a threat for humanity and therefore a number of strategies are adopted to contain that. Many of these strategies are resource driven and requires stringent regulatory commitment. For a country like Bangladesh, where there is inadequate resource and the surveillance system for antimicrobial resistance is ineffective; revival can be a strategy that can be adopted. In the present study, reintroduction of nitrofurantoin was done in patients of uncomplicated urinary tract infection as an alternative to ciprofloxacin and corresponding reversal in sensitivity of urinary isolates was observed after intervention.

The study was designed to determine the trend of sensitivity of urinary isolates against ciprofloxacin. The sensitivity of urinary microbial isolates to ciprofloxacin has increased significantly after reintroduction of nitrofurantoin, which corresponds with the findings of previous studies demonstrating increase in sensitivity after reducing the consumption (Fujita et al., 1994; Austin et al., 1999; Lafaurie et al., 2012). Though, some other studies could not find any strong correlation between the consumption and antimicrobial sensitivity (Bartoloni et al., 2004; Bean et al., 2005; Smith et al., 2008; Bergman et al., 2009).

Couple of studies revealed that the rate of reversal of sensitivity is very slow in community level and is a complex mechanism because reversing the resistance requires restoring the susceptible flora both in human and the environment (Andersson and Hughes, 2010; Barbosa and Levy, 2000).

Interestingly, the increased prescribing of nitrofurantoin after intervention resulted in significantly $(\mathrm{p}<0.05)$ increased sensitivity of urinary microbial isolates to nitrofurantoin after the intervention $(86.3 \%)$ than before $(84.0 \%)$.

Slow development of resistance despite increased consumption was probably due to the diverse mechanism of action of nitrofurantoin (McOsker and Fitzpatrick, 1994), which explained that clinically significant resistance against nitrofurantoin did not develop even after use of the drug for 30 years.

Another objective of this study was to evaluate the clinical outcome of the patients. The clinical cure rate was $80.6 \%$. The finding of the present study suggest that nitrofurantoin could be an effective alternative to ciprofloxacin for empirical treatment of uncomplicated urinary tract infection.

Antimicrobial sensitivity may be revived by reintroduction of older and effective antimicrobial in clinical practice. This revival may allow some time to the scientists to innovate new antimicrobial agent. Revival should be designed on the basis of local sensitivity pattern and need to follow for a definite period. This might be an effective strategy for increasing the sensitivity or delaying the development of resistance and finally containing antimicrobial resistance.

\section{Conclusion}

The study reveals that reintroduction of nitrofurantoin in the treatment of uncomplicated urinary tract infection increases the sensitivity of urinary isolate to ciprofloxacin, suggesting the potential of revival of antimicrobial sensitivity by reintroduction of older and effective antimicrobial.

\section{Financial Support}

Self-funded

\section{Conflict of Interest}

Authors declare no conflict of interest

\section{Acknowledgement}

The authors gratefully acknowledge the support received from Col. Dr. Abdul Quddus Bhuiyan, Clinical Medicine Specialist, Combined Military Hospital, Dhaka, Bangladesh.

\section{References}

Aminov RI. A Brief history of the antibiotic era: Lessons learned and challenges for the future. Front Microbiol. 2010; 134: $1-7$.

Andersson DI, Hughes D. Antibiotic resistance and it's cost: Is it possible to reverse resistance? Nat Rev Microbiol. 2010; 8: 260-71.

Austin DJ, Kristinsson KG, Anderson RM. The relationship between the volume of antimicrobial consumption in human communities and the frequency of resistance. Proc Natl Acad Sci U S A. 1999; 96: 1152-56. 
Barbosa TM, Levy SB. The impact of antibiotic use on resistance development and persistence. Drug Res Updat. 2000; 3: 303-11.

Bartoloni A, Bartalesi F, Mantella A, Amico ED, Roselli M, Strohmeyer M, Barahona HG, Barron VP, Paradisi F, Rossolini GM. High prevalence of acquired antimicrobial resistance unrelated to heavy antimicrobial consumption. J Infect Dis. 2004; 189: 1291-94.

Bean DC, Livermore DM, Papa I, Hall LM. Resistance among Escherichia coli to sulphonamides and other antimicrobials now little used in man. J Antimicrob Chemother. 2005; 56: 962-64.

Bergman M, Nyberg ST, Huovinen P, Paakkari P, Hakanen AJ. Finnish Study Group for Antimicoribal Resistance, 2009. Association between antimicrobial consumption and resistance in Escherichia coli. Antimicrob Agents Chemother. 2009; 53: 912-17.

Cassir N, Rolain JM, Brouqui P. A new strategy to fight antimicrobial resistance: The revival of old antibiotics. Front Microbiol. 2014; 5: 1-15.

Centres for Disease Control and Prevention (CDC). Evidence Supporting the Core Elements. Antibiotic prescribing and use in doctor's offices. Centers for Disease Control and Prevention, Atlanta, Georgia, United States, 2017.

Das AK, Rahman MS. Prescribing vitamins at primary health care level: Exploration of facts, factors and solution. Bangladesh J Pharmacol. 2010; 5: 92-97.

Farrell NR. The landscape of antibiotic resistance. Environ Health Perspect. 2009; 117: 245-50.

Ferdoush J, Ata M, Parveen K, Reza FH, Rahman MS. Knowledge perception and preparedness of future prescribers about antimicrobial stewardship. Bangladesh J Pharmacol. 2016; 11: 928-34.

Fujita K, Murono K, Yoshikawa M, Murai T. Decline of erythromycin resistance of group A streptococci in Japan. Paediatr Infect Dis J. 1994; 13: 1078-78.

Gottesman BS, Carmeli Y, Shitrit P, Chowers M. Impact of quinolone restriction on resistance patterns of Escherichia coli isolated from urine by culture in a community setting. Clin
Infect Dis. 2009; 49: 869-75.

Islam MS, Rahman MS, Misbahuddin M. Impact of prescription audit and feedback on pattern of prophylactic antimicrobials in caesarean section: A cost reduction perspective. Bangladesh J Physiol Pharmacol. 2007: 23: 1-9.

Johora F, Rahman MS. Snapshot of the pharmaceutical promotional literatures of Bangladesh: A critical review. Bangladesh J Pharmacol. 2018; 13: 214-21.

Kraker MEA, Stewardson AJ, Harbarth S. Will 10 million people die a year due to antimicrobial resistance by 2050?. PLos Med. 2016; 13: e1002184.

Lafaurie M, Porcher R, Donay JL, Touratier S, Molina JM. Reduction of fluoroquinolone use is associated with a decrease in methicillin-resistant Staphylococcus aureus and fluoroquinolone-resistant Pseudomonas aeruginosa isolation rates: A 10 year study. J Antimicrob Chemother. 2012; 67: 1010-15.

Laxminarayan R, Duse A, Wattal C, Zaidi AK, Wertheim HF, Sumpradit N, Vlieghe E, Hara GL, Gould IM, Goossens H, Greko C, So AD, Bigdeli M, Tomson G, Woodhouse W, Ombaka E, Peralta AQ, Qamar FN, Mir F, Kariuki S, Bhutta ZA, Coates A, Bergstrom R, Wright GD, Brown ED, Cars O. Antibiotic resistance: The need for global solutions. Lancet Infect Dis. 2013; 13: 1057-98.

McOsker CC, Fitzpatrick PM. Nitrofurantoin: Mechanism of action and implications for resistance development in common uropathogens. J Antimicrob Chemother. 1994; 33: 23-30.

Rahman MS, Begum M, Hoque MZ, Akhter N. Drug Advertisements in medical journals: A commentary. Bangladesh J Physiol Pharmacol. 1999: 15: 31-36.

Sultana SP, Rahman MS. Dynamic online antimicrobial guideline with stewardship program: Impact on antimicrobial prescribing. Bangladesh J Pharmacol. 2017; 12: 364-70.

Smith SP, Manges AR, Riley LW. Temporal changes in the prevalence of community-acquired antimicrobial-resistant urinary tract infection affected by Escherichia coli clonal group composition. Clin Infect Dis. 2008; 46: 689-95. 